\title{
Die Lapplandreise des Seigneur A. de La Motraye i. J. 1718
}

Es ist bekannt, dass der Franzose Jean-François Regnard und der Italiener Francesco Negri, die in der zweiten Hälfte des 17. Jh.s. Lappland besuchten, interessante Reiseberichte hinterlassen haben. ${ }^{1}$ Fast gänzlich unbekannt blieb dagegen die ausführliche Schilderung der Lapplandreise, die Aubry de La Motraye 1718 machte und in seinem umfangreichen Werk über seine grossen Reisen in verschiedenen Teilen Europas und Asiens schon 1723 in London veröffentlichte. ${ }^{2}$ Das ist um so verwunderlicher, als es sich um einen sehr exakten und wahrheitsgetreuen Berichterstatter mit guter Beobachtungsgabe handelt. Erst als 1918 eine schwedische Übersetzung ${ }^{3}$ der Kapitel des 2. Bandes dieses Werkes, die Schweden einschliesslich Lappland betreffen, erschien, wurde es in Schweden bekannt, darüber hinaus aber nur einigen guten Kennern der Reiseliteratur. ${ }^{4}$ Seine ausserordentlich wertvolle und inhaltsreiche Darstellung der Ume-, Lule-, Tornio-, und Kemilappen ist bisher, soweit ich weiss, nicht behandelt worden, trotz des lobenden Urteils K. B. Wiklunds. ${ }^{5}$ Im folgenden sei der Versuch unter-

\footnotetext{
I Siehe E. Itkonen, Regnardin Lapin-kuvaus, Virittājä 1947: 404 -406 und R. Wis, Francesco Negri, voyageur italien du XVII siècle en Laponie et au Cap Nord, Terra Boreale, Helsinki 1969.

2 Travels through Europe, Asia and into part of Africa, Teil 1-2, London 1723.

${ }^{3}$ Seigneur $A$. de la Motrayes resor 1r11-1725, $i$ vilka man finner en stor mängd geografiska, historiska och politiska forskningar över... Turkiet ... Sverige, Lappland etc. . . Urval och översättning av H. Hultenberg. Anmärkningar av S. Bring och K. B. Wiklund. Stockholm 1918. Ubber Kemi-Lappland ist LM's Bericht der älteste überhaupt.

4 Vgl. S. 119, Anm. 1.

${ }^{5}$ In der Einleitung der schwedischen Ausgabe sagt S. E. Bring:
} 
nommen, in einer kurzen Zusammenfassung die wichtigsten Beobachtungen La Motrayes bei den Lappen der betr. Gebiete aufzuzeigen, so dass ersichtlich wird, wie wertvolle Kenntnisse einer alten Volkskultur und alter Volkstraditionen er der Nachwelt erhalten hat.

Die knappen Berichte der einschlägigen Biographien ${ }^{\mathbf{1}}$ bezeichnen als wichtigen Einschnitt im Leben Aubry de La Motrayes (geb. in Frankreich um 1674, gest. in Paris 1743 im folgenden wird sein Name mit dem Sigel LM abgekürzt), dass er als Hugenotte unter Ludwig XIV., der 1685 das Edikt von Nantes aufgehoben hatte, der religösen Unfreiheit wegen i.J. 1695 Frankreich verliess und von da ab als Weltreisender die Mittelmeerländer, den Nahen Osten (einschliesslich der "Tartarei» und der Krim), schliesslich Schweden und danach die Ostseeländer besuchte und sich dann als britischer Untertan in England niederliess. Dort vollendete er sein oben erwähntes Lebenswerk, dem andere Schriften folgten. ${ }^{2}$ Mit seinen Freunden in Schweden blieb er in Verbindung.

Vad slutligen de la Motrayes resa till Lappland angår, har professor K. B. Wiklund om denna uttalat, att den "är en av de märkligaste, som i äldre tid företagits. Resenären skyr inga strapatser och är icke rädd för att bege sig ut i alldeles okända trakter och på närmaste håll taga kännedom om lapparnas liv. Han har ständigt ögonen med sig, ser på tingen med nykter blick och nedskriver sina iakttagelser på ett klart och interesseväckande sätt. Hans reseskildring ār därför också en av våra bästa källor för kunskapen om lapparna i äldre tid, och det är blott att förvåna sig över, att den hittills blivit så litet uppmärksammad och begagnad». Die erste Erwähnung durch K. B. Wiklund steht in Svenska Turistföreningens årsskrift 1904: $359 \mathrm{ff}$.

1 Siehe u.a. La France littéraire ou Dictionnaire Bibliographique par J. M. Quérard, Bd, 4, Paris 1830, S. 510 und Bonniers Lexikon 8: 1134, Stockholm 1964. Die von S. E. Bring vorgezogene Jahreszahl 1747 pro 1743 als Todesjahr scheint nicht so glaubwürdig.

2 Der englischen UUbersetzung (Travels ...) folgte die Herausgabe des französischen Originals Voyages en Europe, Asie \&. Afrique, ou l'on trouve une grande parieté de recherches geographiques, historiques \& politiques sur l'Italie, la Grece, la Turquie, la Tartarie crimée \& nogaye, la Circassie, la Suede, la Laponie etc. . . T. 1-2. Fol. La Haye 1727. Dieses französische Original legen wir den folgenden Ausführungen zugrunde. Die englische Erstausgabe lernte ich vor etlichen Jahren in der Universitätsbibliothek Göttingen kennen. Eine auf deutsch i.J. 
Den Anfang seiner Reisen bildete ein 6monatiger Aufenthalt in Rom, wo vermutlich sein Interesse für Beziehungen zu ausländischen Diplomaten erwachte, die er dann in einer Zeit politischer Hochspannung in Konstantinopel eifrig betätigte. Seine Berichte über die Vorgänge in der Türkei seit dem Frieden von Karlowitz (1699) bis zu seiner Begegnung mit dem in die Türkei geflohenen schwedischen König Karl XII. (in Bender 1711) werden von den Historikern als zuverlässige Quelle waus erster Hand» angeführt. Seine Geschicklichkeit, sich als Augenzeuge Kenntnisse zu verschaffen, zeigt u.a. die Art, wie es ihm gelang, als Türke verkleidet in Begleitung eines französischen Uhrmachers in den Serail und sogar den Harem des Sultans einzudringen. Aus der Türkei begab LM sich nach einem kurzen Aufenthalt in Norddeutschland im Herbst 1715 nach Schweden, wo er sich bis 1720, reichlich 5 Jahre, aufhielt. Während dieses Schwedenaufenthalts machte er im AprilJuli 1718 seine Reise nach Lappland (Voyages en Europe... 1727, Bd. II, Fol., Kapitel 16-17, S. 329-391). Er schrieb regelmässig Aufzeichnungen in sein Tagebuch, und in Tagebuchform ist auch sein Reisebericht gehalten.

\section{Der Verlauf der Reise}

Am 7. April 1718 brach LM in Begleitung O. V. Klingströms, eines Beamten der Kgl. Kanzlei, aus Stockholm auf. Der Weg führte ihn auf Umwegen nach Umeå (Ankunft am 6. Mai), von wo er zu Pferd nach Luleå ritt. Teils mit Schlitten, teils

1783 im Auszug erschienene Ausgabe behandelt hauptsächlich den Aufenthalt Karls XII. in der Türkei. Eine Fortsetzung der Reiseberichte, die das Jahr 1725 betreffen, bildet Voyages en Anglois et en François en diverses provinces et places de la Prusse ducale et royale, de la Russie, de la Pologne etc., contenant un traité de divers ordres de chesalerie... La Haye 1732. In seiner Bewunderung für Karl XII. nahm er kritische Stellung zu Voltaires Histoire de Charles XII in der Schrift Remarques historiques et critiques sur l'Histoire de Charles XII, roy de Suède par' $M$. de Voltaire, pour servir de supplement à cet ouvrage, die u.a. auf italienisch und spanisch erschien. 
zu Fuss kam er nach Raine, das ihm als Geburtsort des "bis heut bewunderten Abenteurers» Nicolaus Örn ${ }^{1}$ bekannt war. Von dort setzte er seine Wanderung zum Kalix und nach Tornio (Ankunft am 19. Mai) fort. ${ }^{2}$ Erst als das Eis schmolz, konnte er im Boot mit einen Dolmetscher und zwei (finnischen) Ruderern sein Ziel den Torniojoki aufwärts weiter verfolgen, musste aber wegen der starken Gegenströmung zu Fuss von Yli-Tornio nach Kengis wandern. ${ }^{3}$ Mutig setzte er den Weg zu seinem Reiseziel, dem Tornio-See fort. Bei Junosuanto sah er zum ersten Mal Lappenzelte, und zwar zunächst die im Wald zurückgelassenen leeren Gerüste, die den für den Sommer in die Fjelde Norwegens mit ihren Rentieren gezogenen Lappen gehörten. Aber bald darauf traf er in derselben Gegend Lappen, die in dauerhafteren Zelten wohnten, offensichtlich Waldlappen, die es dort schon lange nicht mehr gibt. LM studierte ihre Wohn- und Lebensweise ganz genau. Und nunmehr vergeht kaum ein Tag, an dem der unermüdliche Reisende nicht irgendeine Lappenfamilie, oft mehrere an einem Tag besucht.

Auf seiner Route liegt Svappavaara, zu dessen Erzhütte er auf einem Floss den Stamp stromaufwärts gelangt, ferner die nahe aufeinander folgenden Seen Luspa-, Sautaus-, und Jukkasjärvi. Überall findet er dort fischende Lappen und Finnen. Das Kirchdorf Jukkasjärvi, bei dem er am 6. Juni ankommt, wird zu einem wichtigen Anfangs- und Endpunkt seiner Wanderungen und Fahrten um und über den Tornio-See.

1 N. Örn, Verfasser des unbedeutenden Büchleins Kurtze Beschreibung des Lapplandes, Bremen 1707, durchreiste in den Jahren 1702 und 1703 viele Länder West- und Südeuropas - er trat in Lappentracht und auf einem Rentier reitend als „Fürst von Lappland» u.a. vor dem Hof in Hannover auf - und landete schliesslich in Astrachan (1710), wo er nach längerer Gefangenschaft starb.

${ }^{2}$ Der Anblick der durch den Russeneinfall drei Jahre früher grösstenteils zerstörten Stadt veranlasste ihn, in sein Tagebuch den Vers einzutragen: Nunc seges est ubi Troja fuit, - Anspielung auf O. Rudbecks Atlantica I, 36.

${ }^{3}$ LM erwähnt, dass der Bischof Joh. Bilberg dort 1695 naturwissenschaftliche Beobachtungen (deren Resultat die ihm bekannte Schrift Refractio solis inoccidui, in septentrionalibus oris detecta... war) gemacht, aber nicht gewagt hatte, weiter ins Land vorzudringen. 


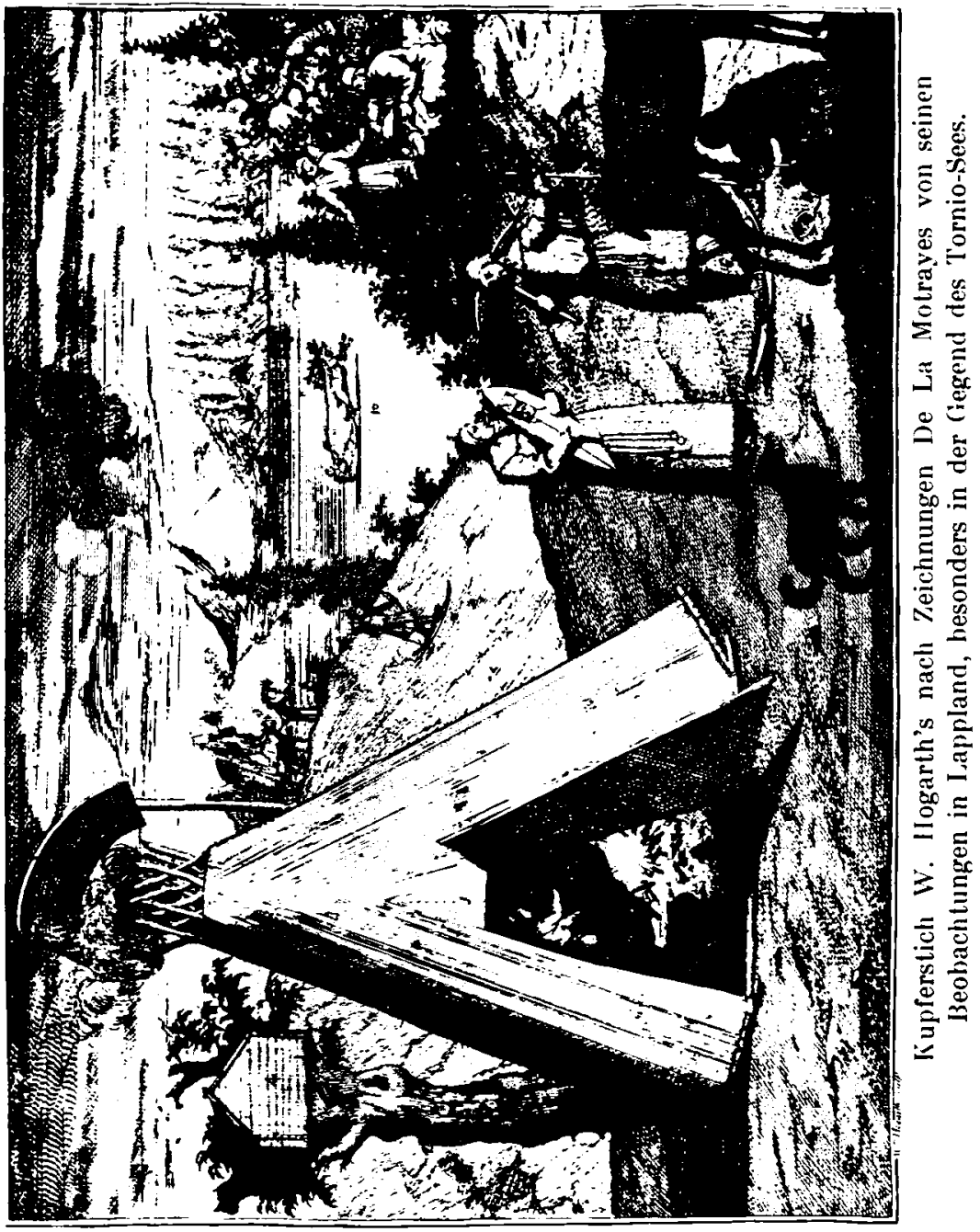


Hier erhält er einen neuen Dolmetscher, einen ausgezeichneten Kenner und Freund der Lappen, der hier wohnhaft ist. An den Abhängen der umliegenden Berge, von denen wir Namen wie Oppivaara, Rodevaara und Mickavaara erfahren, hat LM nun besonders interessante Begegnungen und Gespräche mit verschiedenen Lappen und ihren Familien. Wenn seine Wegführer oder Gastgeber schlafen, steigt er um Mitternacht auf die Höhen und betrachtet, Pfeife rauchend, den Stand der Sonne. Er versteht sich mit ihnen so gut, dass es ihm gelingt, einen 70jährigen Lappen als Führer für eine Schlittenfahrt mit Rentieren auf dem Torniojärvi zu gewinnen.

Das Glück will, dass derselbe Lappe vor nahezu 37 Jahren drei Franzosen, darunter den durch seine Voyage de Laponie berühmt gewordenen Lustspieldichter Jean-François Regnard, zu dem auf der Spitze einer Halbinsel des Sees gelegenen Endziel ihrer Reise geführt hatte. Der greise Lappe, der sich genau daran erinnert, bringt nun LM auf der Schlittenfahrt zu dieser Landzunge. An der Spitze der Lindzunge macht LM am Ufer eine geradezu sensationelle Entdeckung. Als der Lappe dort eine Stelle zeigt, wo er Regnard auf einen Stein etwas hatte einritzen sehen, kratzt L,M das über den Stein gewachsene Moos weg und findet eine Inschrift, die er in seinem Tagebuch aufzeichnet. Das geschah am 8. oder 9. Juni 1718. Später, etwa am 22. Juni, sah er die Kopie dieser Inschrift, die ebenso wie die auf den Stein eingeritzte von Regnard und seinen Gefährten stammt, in der Kirche von Jukkasjärvi. Mit diesem Bericht de La Motrayes findet ein Rätsel, das die Forscher bis in die Gegenwart beschäftigt hat, seine Lösung.

Regnard erzählt in seinem Reisebericht, der zum ersten Mal 1731 und erneut 1875 gedruckt wurde, und in dem der Wortlaut der Inschrift steht, dass er sich in Jukkasjärvi mit seinen Gefahrten anderthalb Tage abgemüht hat, die Inschrift in einen Stein, den sie dann zu dem bestimmten Berg mitgenommen hătten, einzugraben ${ }^{1}$ und dass

1 Voyage de Laponie, Paris 1875, S. 67: „Nous fúmes occupés le rest de ce jour et toute la matinée du mardi, à graver sur une pierre des monuments éternels, qui devoient faire connoître à la postérité que trois François n'avoient cessé de voyager q'où la terre leur avoit manqué... und 
sie eine Kopie davon in eine Holztafel einritzten, die später im Altarraum der Kirche an der Wand angebracht wurde. Die Holztafel konnte zwar nachgewiesen werden (zuerst von J. W. Zetterstedt in Resa genom Sweriges och Norriges lappmarker, förrättad år 1821, Lund 1822, S. 230 und in neuester Zeit durch photographische Wiedergaben; vgl. auch E. Itkonen op. cit. 1947), aber von dem Stein war keine Spur zu entdecken. Deshalb glaubte man allgemein, dass die Behauptung Regnards von der Aufstellung des Steins reine Erfindung war. Erst nach Bekanntwerden des Reiseberichts LM's Anfang dieses Jahrhunderts in Schweden fand man darin das Zeugnis dafür, dass der "Gedenkstein" tatsāchlich existiert hatte. ${ }^{1}$ Da LM's Reisebericht 1723, der Regnards aber erst 1731 im Druck erschienen, hātte LM ein Hellseher sein müssen, um von dem Stein Regnards erzählen zu können, ohne ihn gesehen zu haben. In einigen Details bestehen dennoch Unklarheiten. Nur bei LM findet sich unter den Versen der Inschrift und den Namen der drei Franzosen die Ortsbezeichnung Ad Pescomarcam. Bei Regnard kommt dieser Name überhaupt nicht vor, statt dessen gibt er dem Berg an der Spitze der Halbinsel den selbsterfundenen Namen Metavara. Nach K. B. Wiklund hatte die Halbinsel den lappischen Namen pieskénjārka (a.a.0. 1904: 362). Da LM den Namen Pescomarca zur Bezeichnung der Halbinsel an verschiedenen Stellen seines Reiseberichtes gebraucht, hat er wohl den lappischen undeutlich gehörten Namen auf seine Weise wiedergegeben und in seiner sonstigen Genauigkeit eigenmächtig unter die Inschrift Regnards gesetzt.

Von dieser in ost-westlicher Richtung in den see sich erstreckenden Halbinsel aus setzt LM seine Schlittenfahrten in

S. 95: "Nous avançâmes bien sept ou huit lieues dans le lac, proche une montagne qui surpassoit toutes les autres en hauteur... Ce fut là que nous plantâmes l'inscription précédente, qui étoit sa véritable place...."

1 Diese Auffassung bestätigen H. Kjellman: Regnards resa $i$ Lappland in Svenska Turistföreningens årsskrift 1931: 235-258, R. Wis: Francesco Negri, voyageur italien du XVII siècle en Laponie in Terra Boreale, Helsinki 1969, S. 48 Anm. 14 und S. 44 Anm. 5 (gegen die Zweifel L. Maury's) wie auch A. G. Elg: Inskriften vid 'Metavara' in Fornvännen 43, 1948: 52-54, wo er sagt: 'Resultat av denna textkritiska granskning skulle alltså bli, att... den äkta lydelsen återfinnes på minnestavlan i Jukkasjärvi kyrka samt hos La Motraye, vilka måste anses utgöra våra primära källor».

${ }^{2}$ A. J. Elg (op. cit.) geht in seiner Gläubigkeit an die Wahrheitstreue LM's vielleicht $z u$ weit, wenn er "Ad Pescomarcam" nicht als Zusatz, sondern als Teil des Originals der Inschrift ansehen will. An dieser Stelle sei es mir gestattet, dem Bibliothekar Herrn Dr. Henrik Grön. roos meinen herzlichen Dank für viele Quellenhinweise auszusprechen. 


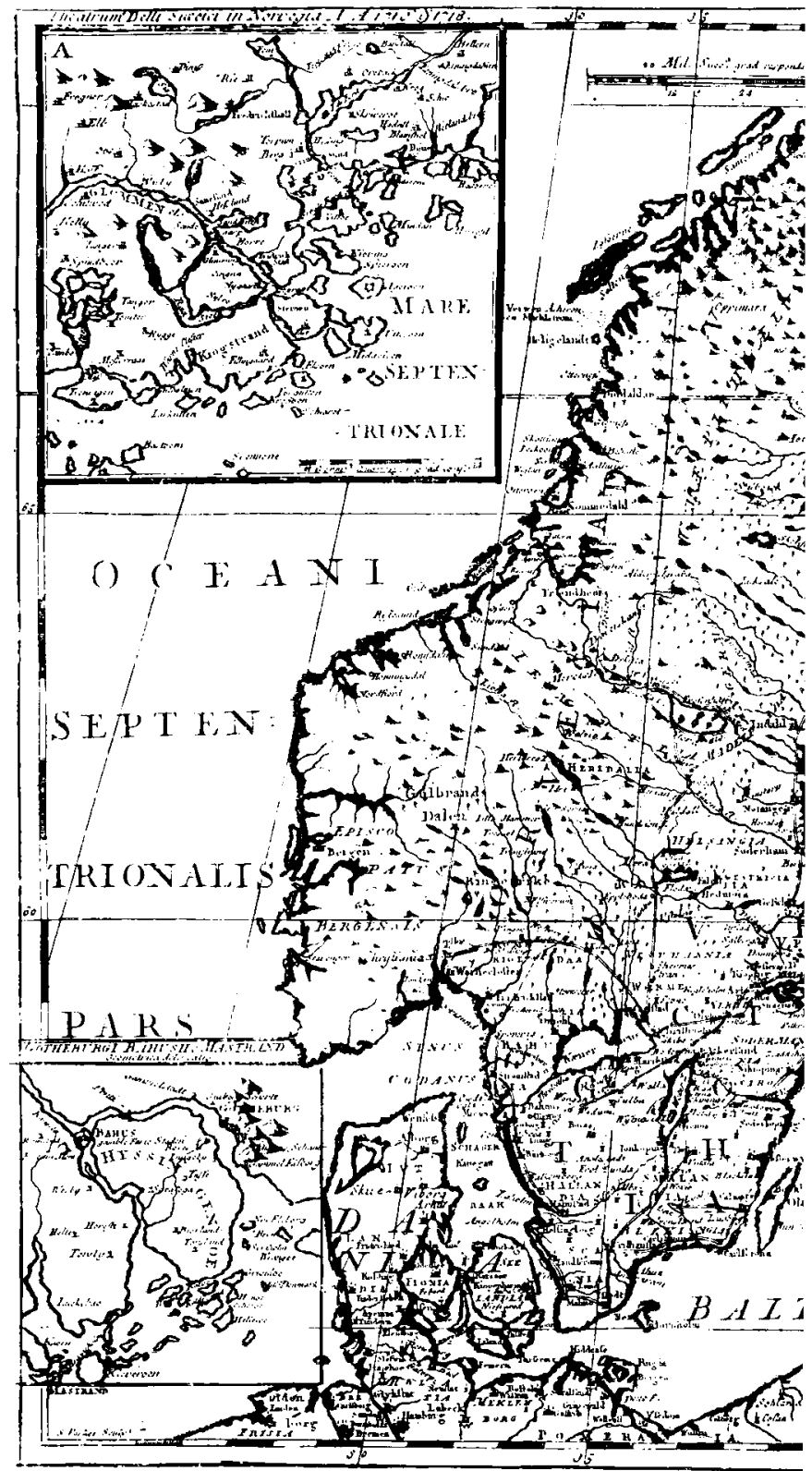

De la Motrayes Karte von Skar wegs nach Lappland $b$ 


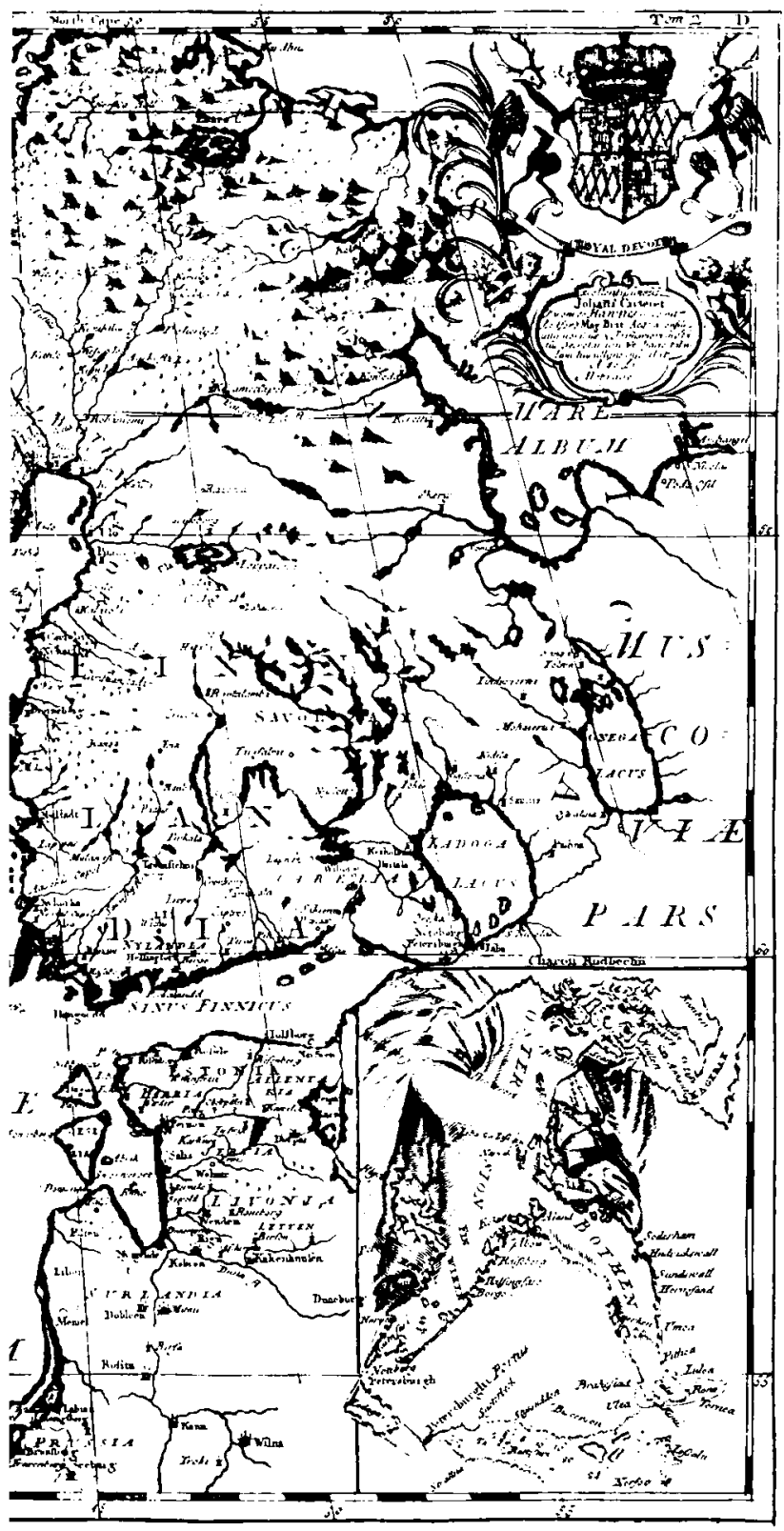

it Einzeichnung seines Reiselio- und Kemijärvi. 
nördlicher Richtung fort und begibt sich auf Wanderungen in den norwegischen Fjelden. Von den Höhen aus sieht er das Eismeer und glaubt bei dessen majestätischem Anblick mit mehr Recht als Regnard sagen zu können, dass der Erdball hier für seine Augen die Grenze des Unendlichen berührte. Den letzten Höhepunkt erreichte LM's Lapplandreise in den norwegischen Bergen am Torniojärvi bei der Begegnung mit einem lappischen Zauberer ("Magicien"), die ihm endlich nach vielen Bemühungen - er wollte dem schwedischen König, der durch seine Beamten vergeblich nach Zauberern hatte suchen lassen, mit der Nachricht von der Begegnung eine Überraschung bereiten --- durch eine List gelang. Ein lappischer Rentierhirt führte ihn heimlich zu einem mit seiner Familie in ärmlichen Verhältnissen lebenden Lappen, der sich auf die magische Kunst, die er mit Hilfe seiner Trommel nach alten Traditionen ausübte, verstand.

Nach der Rückwanderung am Ostufer des Sees, der wegen des Treibeises nicht befahrbar war, kam er am 22. Juni wieder in Jukkasjärvi an. Auf Vorschlag des Pfarrers schrieb auch er auf eine Holzplatte eine inzwischen verlorengegangene Inschrift, die ich im folgenden nach der Kopie von J. W. Zetterstedt $^{1}$ im originalen Wortlaut - LM hat die Hexameter später für den Druck geglättet - wiedergebe:

Gallia mi' lucem dedit, et liberum Anglia portum, Utraque me Germania, Graecia magna minorque, Mons Jovis, ac Barcellona Herculeaeque Columnae, Ambae Asiae, Italia, Africa. Hisque opposita Melite, Euxina et Moeotica, Caspia, Baltica nec non

Littora viderunt, vidit polus arcticus ipse.

Et mihi inocciduum ostendit Lapponia solem,

1 Zetterstedt fand und kopierte (op.cit. S. 230-234) in der Kirche von Jukkasjärvi im ganzen 13 Inschriften von Lapplandreisenden. Unter diesen befanden sich G. Gyllengrijp, J. Wegelius, der polnische Fürst Josephus Eusebius Jablonowski (seine Inschrift vom 12. Februar 1804 endet mit den Worten: "Dum peregrinans totam vidi Europam - Ut etiam independentes vidissem Sameos - Laponiam adveni»), verschiedene Franzosen, Italiener und Engländer. Im Datum LM's ist offensichtlich ein Schreibfehler passiert. Es muss heissen: 23. Jun. 
Proque cibo et potu carnem ac lac rangiferinum

Praebuit, ut Tartaria olim praebebat equinum.

Haec scribebat ad Juckasjervam

Rediens ex Tornaviensi Lacu

Aubry de la Motraye

Magnae Britanniae Subditus.

\section{Jun. 1718}

Einen neuen Abschnitt seines Reisewegs bildet sein Aufenthalt in Kemi-Lappland (26. 6.-3. 7. 1718). Von Tornio aus fährt er im Boot nach dem vier Meilen entfernten Kirchdorf Kemi, das oberhalb der Mündung des Kemijoki an dessen westlichem Ufer liegt. Von der einst volkreichen Ortschaft mit aufblühendem Handel war nichts erhalten geblieben als die mittelalterliche Steinkirche (bâtie en Croix Latine), neben der sich der Pfarrer als einziger Einwohner am Ort in dem abgebrannten Pfarrhaus notdürftig eingerichtet hatte. Von dessen gerade hier weilendem Sohn Zacharias Forbus, dem künftigen Pfarrer von Kuusamo, der ganz Kemi-Lappland sehr gut kannte, erhält LM wichtige Schilderungen des Gebiets und der darin wohnenden Finnen und Lappen, die er uns in seinem Reisebericht mitteilt. Er selbst kommt im Boot den Kemijoki stromaufwärts an Lapinniemi (Tervola) vorbei nach Rovaniemi, wo er sich auf dem Markt Pfeil und Bogen der Lappen zum Andenken kauft, und zum Kemijärvi. "An dessen Ufern rings um den ganzen See sah ich so viele Lappen, dass ich mir über die anderen in dieser Lappmark ein Urteil bilden kann.»

Die Rückreise machte LM von Kemi aus im Boot bis Kalix, von dort zu Pferd über Råneå nach Luleå, wo er den Luleälven aufwärts einen Abstecher in die Gegend von Jokkmokk machte, um in einem kleinen Flusse Perlmuscheln zu fischen. Da der Wasserstand um diese Jahreszeit (Anfang Juli) zu hoch war, musste er sich mit der Beschreibung der zu erbeutenden Perlen begnügen. In der Nähe des Flusses macht er noch einmal interessante Beobachtungen bei Lappen. Von Luleå ritt er dann nach Gamla Piteå, wo er den 21jährigen Per Fjellström traf, der im folgenden Jahr Lehrer an der Lappenschule 
in Lycksele ${ }^{1}$ werden sollte und ihm begeistert von der Lappenschule und den Pitelappen erzählte. Die letzte Station seiner Lapplandreise war Umeå, wo er sich am 13. Juli vom Gouverneur verabschiedete.

Dem Reisebericht fügte LM eine Karte Skandinaviens bei (im 2. Teil seiner Travels...1723 und Voyages...1727, Karte D), die er mit Hilfe von Freunden in Schweden ${ }^{2}$ mit Einzeichnung seiner Reiseroute und der von ihm besuchten Orte u.a. in Tornio- und Kemilappland durch S. Parker anfertigen liess und Lord John Carteret ${ }^{3}$ widmete.

Die Karte ist in Anbetracht der frühen Zeit und ihrer Originalität wertvoll und verdient schon wegen der Darstellung Lapplands und Finnlands publiziert zu werden. Es muss auf einem Versehen oder auf Oberflächlichkeit der Leser beruhen, dass sie bisher nicht bemerkt und leider auch in der schwedischen Ausgabe dieses Teils der Voyages nicht einmal erwähnt wurde. ${ }^{4}$

Lebensweise, Sitten, Religion und Kultur der L a p p n

Mit guter Beobachtungsgabe studierte LM die Lebensweise der Lappen. Bald nachdem er in den Wäldern von Junosuanto die leeren Gerüste der Zelte der in die norwegischen Fjelde gewanderten Lappen gesehen hatte, konnte er am Fusse des Siikavaara zwei bewohnte Zelte von Waldlappen kennenler-

I Diese Lappenschule, die 1617 in Piteå gegründet worden war, stand seit der Übersiedlung nach Lycksele unter der Leitung des Reichsrats Johan Skytte.

2 Siehe seinen Brief an Erik Benzelius vom 13. Januar 1723 aus London, Hschr. der Stifts- och landsbiblioteket i Linköping.

3 Über die Tätigkeit Carterets als Gesandter in Schweden siehe H. Grönroos, England, Sverige och Ryssland 1719-1721 in Historisk Tidskrift för Finland 16, 1931, S. 1-26 und 57-66.

4 Das in der rechten unteren Ecke der Karte eingefügte Bild *Charon Rudbechii» hat LM der Schrift O. Rudbecks d. J. Nova Samolad sive Lapponia Illustrata, Upsalae 1701, S. 36 entnommen. 
nen. Von dem ersten Zelt gibt er eine genaue Schilderung und eine Zeichnung auf dem kombinationsreichen Kupferstich ${ }^{1}$, der der englischen und französischen Ausgabe beigefügt ist. Das Zelt ("Cotta») ist aus hohen Stangen in der Weise errichtet, dass die Spitzen sich oben genügend weit überkreuzen. Zwischen den Stangen sind Wände durch zusammengebundene Zweige gebildet (was für einen Zelttyp der Waldlappen charakteristisch ist -- siehe Scheffer Lapponia) und von aussen mit grobem Tuch überdeckt. Die Dauerhaftigkeit des Baues wird durch Querstützen am Boden gefestigt. Originell ist das im Bedarfsfall an einer hohen Stange aufstellbare Schutzdach über dem Rauchloch, dessen Masse und konvexe Form genau beschrieben werden (Abb. Nr. 2). Die Rentierfelle als Lager und Bedeckung für die in puris naturalibus schlafenden die Reichen haben auch Matratzen, die mit Rentierhaaren, und Kopfkissen, die mit Federn gefüllt sind -- und als Kleidung gefallen LM so sehr, dass er sich schon nach einigen Tagen wie ein Lappe in Pelz und Lappenmütze kleidet (ähnlich wie C. Linné auf seiner Lapplandreise 1732). Bei der Beschreibung der lappischen Frauentracht erwähnt er die engen langen Hosen ("Paussa", sprich pavsah), wie sie auch in Ungarn (das er durchreist hat) getragen werden. Als Kopfbedeckung tragen die Frauen jetzt im Sommer eine aus rotem Tuch angefertigte, brodierte Mütze. Vom Frauenschmuck beschreibt er u.a. den fast handbreiten "in sehr sinnreicher Weise» mit Zinnplatten bedeckten Ledergürtel.

Als LM später in Oppivaara eine bourse brodée in einem Zelt hängen sah und sich für die lappischen Handarbeiten interessiert zeigte, brachte die Lappin ihm eine ganze Truhe voll

${ }^{1}$ Das von dem später berühmt gewordenen Kupferstecher W. Hogarth nach den Anweisungen LM's angefertigte Bild enthält volkskundlich interessante und, abgesehen von einigen Missverständnissen (u.a. Befestigung des Zügels an den Hörnern des Rentiers Abb. Nr. 10, zu offenes Melkgefäss Abb. Nr. 8) und einigen Zusätzen des Künstlers (Zweige um den auf dem Baumstumpf befestigten Vorratsspeicher Abb. Nr. 1) zuverlässige Darstellungen der im Text geschilderten Gegenstände. Dem Assistenten am Institut für Volkskunde a.d. Universität Helsinki, Herrn HuK Samuli Aikio danke ich für freundl. Beratung. 
Kleidungs- und Schmuckstücken herbei. Grossen Eindruck machte auf ihn die Art, wie die Lappinnen zum Nähen für ihre Broderien Zinn (und Blei) durch eine mit Löchern verschiedener Grösse versehene Platte ${ }^{\mathbf{1}}$ aus Knochen (?Horn) des Rens mit den Zähnen zu Fäden zogen (Abb. Nr. 3 - im Zelt). Diesen Brauch beobachtete LM noch einmal bei seiner Rückkehr am Luleälven. Er vergisst nicht, die Sauberkeit der Lappen zu erwähnen, weil er verschiedentlich Lappen sah, die am Morgen ihren ganzen Körper im Schnee wuschen.

Die Mahlzeiten werden gewöhnlich in Holzschüsseln und auf Holztellern serviert. Es gibt meistens Rentierfleisch (warm oder kalt, oft aus dem Vorrat des getrockneten - ein besonderer Leckerbissen ist Rentierzunge), Fisch und Käse. Dazu wird Rentiermilch als Getränk gegeben. Verglichen mit der Pferdemilch der Kalmücken fand $\mathrm{LM}$ die frische Rentiermilch ${ }^{2}$ wohlschmeckend und von angenehmem Duft, der von den Kräutern Lapplands, besonders von der Angelica archangelica herrühre. Brot gibt es nicht ${ }^{3}$ (das Wort dafür haben die Lappen aus dem Finnischen entlehnt). Als Ersatz bereiteten die Lappen aus Milch und Gerste- oder Hafermehl, das sie von den Norwegern kauften, eine Art Brei. Tabak ist Luxus. Statt dessen kauen die Lappen, auch die Frauen, die Wurzel der Angelica archangelica, die dem Atem einen angenehmen Geruch verleiht. Überhaupt ist LM von der Esskultur und Gastfreundlichkeit der Lappen sehr günstig beeindruckt und hebt hervor, dass die Mahlzeiten wegen der lebhaften und fröhlichen Natur der Lappen mit sehr gutem Humor gewürzt waren. Ganz grossartig war die Bewirtung in Rodevaara, wo das Fleisch

1 Vgl. T. I. Itkonen, Suomen lappalaiset I, Helsinki 1948, S. 345, Abb. 158: 4.

${ }^{2}$ Am Luleälven sah LM, wie ein Lappenmädchen Rentiermilch in einem Holzbottich mit zwei Stöcken, die wie Trommelschlegel aussahen, butterte.

${ }^{3}$ In der Gegend von Tornio sah er die häufige Wasserpflanze Calla palustris (Linné, Flora Lapponica, 1737, Nr. 320) und schildert, wie die einheimische Bevölkerung aus der Wurzel Brot bereitet ("missnebröd", vgl. C. Linné, Berättelse Om The Inhemska växter, som $i$ brist af Säd kunna anwändas til Bröd- och Matredning, 1757, Nr. 7. Calla palustris). 
eines eigens für ihn geschlachteten jungen Rentieres am Spiess gebraten und das reichhaltige Mahl mit silbernen Löffeln gegessen und die Milch in einem grossen Silbergefäss geboten wurde.

Unser Lapplandreisender schloss schnell Freundschaft mit den Lappen ", die ihm gar nicht klein und hässlich vorkamen. Er schildert sie als wohlgewachsen, mittelgross, mit frischer roter Hautfarbe: "Wenn ich diese Lappen», so schreibt er, "mit dem verglich, was ich in verschiedenen Reiseschilderungen gelesen habe, in denen sie als Pygmäen dargestellt werden, fing ich an, meine Vorstellungen von ihnen zu berichtigen.»

Ihren Lebenserwerb erhalten sie durch Rentierzucht, aber auch durch Fischen (Lachse und Renke -- finn. siika) und Jagen von Pelztieren, Wildbret und Vögeln. Einige Lappen sind wohlhabend, mit Rentierherden von $700-800$ Stück, andere besitzen kaum 7 Rentiere und verdingen sich in Lohnarbeit bei anderen Lappen. Als LM in Oppivaara eine Lappin fragte, ob sie ihrer heiratsfähigen Tochter Rentiere in die Ehe mitgebe, antwortete sie: Bei uns bekommt jedes Kind gleich bei seiner Geburt einige Rentierkühe, aber auch Stiere zum Geschenk, so dass bis zu seinem heiratsfähigen Alter eine ganze Herde herangewachsen ist. ${ }^{2}$

Die Lebensweise der Kemilappen ist von denen der Südlappen recht verschieden. Sie kleiden sich vielfach ähnlich wie die dort lebenden finnischen Bauern und Fischer, wohnen meistens in festen, mit Baumrinde oder Torf bedeckten Hütten, die in den südlichen Teilen von Kemi-Lappland auch einen Herd haben. Ihre Siedlungen bilden kleine Dörfer. Sie halten auch Vieh (Pferde, Ochsen, Kühe und Ziegen), für die sie (nach LM) jäliälä als Winterfutter sammeln. Eine Hauptrolle im Lebenserwerb spielt der im fischreichen Kemijoki besonders ergiebige Lachsfang, aber auch die Jagd auf Pelztiere, unter denen der im Torniogebiet fast ausgestorbene Schwarzfuchs

1 Davon zeugen die zahlreichen Andenken, die er zum Geschenk erhielt, u.a. einen rassigen Wachhund (Abb. Nr. 14).

${ }^{2}$ LM bestätigt, was er schon bei Scheffer gelesen hatte, dass der Bräutigam den Eltern der Braut Geschenke bringt. 
und der Biber zu nemnen sind. Ausserdem gibt es hier wildo Rentiere, die mit Pfeil und Bogen gejagt werden. Im übrigen gebrauchen die Kemilappen wie die Finnen Feuerwaffen.

Am Ende seiner Lapplandreise fasst I,M seine Eindrücke von den Lappen zusammen und bewundert vor allem ihren Fleiss und ihre Geschicklichkeit in den Heimarbeiten: Sie haben keine anderen Arbeitsgeräte als eine Axt und ein spitzes Messer, aber es gibt keinen Lappen, der nicht selbst sein Boot, Fischnetz, Schlitten, Skier usw., mit allem was dazu gehört, Pfeil und Bogen wie auch die Hausgeräte anfertigt. Die Frauen sind ausserordentlich geschickt in der Bearbeitung von Leder, Fellen, Haut, Knochen, Horn und Sehnen wie auch der genannten Metalle und des Holzes (u.a. Wurzeln) zur Herstellung der Kleidungsstücke und des Zierats (Broderien). Sie fühlen sich zufrieden und glücklich in ihrer Lebensweise, die sie mit keiner anderen vertauschen möchten. ${ }^{1}$ Sie gibt ihnen ein beneidenswertes Gefühl der Freiheit und Sorglosigkeit, und sie vertrauen sich den Gesetzen der Natur an, die sie befolgen. Deshalb sind die Lappenfrauen auch treue Gattinnen.

LM's Lieblingsthema, über das er mit den Lappen immer wieder ins Gespräch zu kommen suchte, war die Religion. Er konnte aber nur grosse religiöse Unwissenheit bei ihnen konstatieren und kam enttäuscht zu der Feststellung, dass die Religion für sie eigentlich nur in der Leistung der pflichtmässigen Abgaben, dem jährlichen Kirchenbesuch und den äusseren Formen von Taufe, Trauung und Begräbnis bestand. Da er bei jeder Gelegenheit nach lappischen Zauberern fragte, be-

1 Davon konnte sich LM nicht bloss durch die Antworten auf seine z.T. scherzhaften Fragen (u.a. in Rodevaara die scherzhaft gemeinte Frage, ob die Eltern ihm nicht ihre Tochter zur Frau mitgeben möchten) überzeugen, sondern auch als er auf der Rückreise in Söderhamn zwei Lappenburschen und ein Mädchen in festlicher Tracht traf, die Fredrik I, damals noch Erbprinz, zu seinem Vater, dem Landgraf Karl von Hessen-Kassel als Sehenswürdigkeit geschickt hatte. "Sie gaben mir zu verstehen, dass sie ihre Wälder, Felsen, Seen und Berge, in die sie nun zurückkehrten, allen Städten und Dörfern in Schweden und Deutschland und all den Bequemlichkeiten, die man ihnen dort bieten könne, vorzōgen.» 
merkte er ihre Furcht vor Unglück und Krankheit und sehr viel Aberglauben. Deshalb, und auch aus Furcht vor der Regierung, gaben sie ihm immer wieder die Antwort, dass es keine Zauberer in Lappland mehr gebe. Als ihm endlich die oben geschilderte Begegnung mit einem zauberkundigen Lappen gelang, machte er genaue Beobachtungen, deren Schilderung sehr bemerkenswert ist (Abb. Nr. 9):

Die Trommel, die der $\mathrm{N}$ o id aus einem Versteck holte, war in der Form einer Kesselpauke oder dem Schallkörper einer Laute ähnlich ${ }^{1}$. Das auf die ovale Schale gespannte, durchsichtige und mit Figuren bemalte Fell diente, wie sich bald zeigen sollte, nicht bloss zum Trommeln, sondern auch zum Orakeln. Am Boden des kesselförmigen Rumpfes aus Holz hingen am Griff in der Mitte an Ketten eine Reihe metallener Ringe. Links und rechts rom Griff befand sich im Holz je eine 8 Zoll lange und 1 Zoll breite Öffnung. Durch diese liess der Zauberer die Ringe, ehe er das Trommeln begann, hineinfallen, so dass sie, an den Ketten hängend, auf dem Trommelfell zu liegen kamen. Zum Trommeln diente ihm als Schlegel ein Y-förmiger Hammer aus einem Stück Rentiergeweih.

Beim Trommeln hielt er das Gerät am Griff in der Hand, mit der Membrane nach unten, und schlug mit dem Hammer von unten nach oben aufs Fell, so dass die Ringe darauf zu tanzen begannen und ein klapperndes Geräusch verursachten, ähnlich dem eines Tamburins. Das dauerte einige Minuten. Dann legte der Zauberer sich auf den Rücken (nicht auf den Bauch, wie Reisebeschreibungen sagen!) und senkte die Trommel vorsichtig, ohne sie umzuwenden oder nach den Seiten zu neigen auf seinen entblössten Bauch, schloss die Augen, schien in Ohnmacht zu fallen und nicht mehr zu atmen. In dieser Stellung verharrte er eine Stunde. Dann hob er die Trommel mit

\footnotetext{
1 Sie gehörte also zum Typ der Schalentrommeln. Siehe u.a. E. Manker, Die lappische Zaubertrommel, Nordiska Museet: Acta Lapponica, I, Stockholm 1938, V. Kap. S. 151 ff. Beachtenswert ist LM's Bemerkung, dass er eine ähnliche Trommel selbst besitze, d.h. nach London mitgenommen habe. Vgl. bei Manker (op. cit. S. 752-753) die Trommel Nr. 56 (British Museum, Dep. of Ethn., London) mit LM's Beschreibung.
} 
beiden Händen, ohne sie im geringsten zu neigen oder zu schütteln, nahe vor seine Augen und betrachtete aufmerksam durch das Trommelfell die Lage der in der Trommel befindlichen Ringe zu den aufgezeichneten Figuren und begann seine Voraussagungen zu sprechen. ${ }^{1}$

LM fand die Prophezeiungen so allgemein gehalten, wie es für Wahrsager typisch ist, dass sie auf ihn keinen Eindruck machten. Als er anfing, den Noid über sich selbst auszufragen, wer er sei usw., war aus diesem nichts herauszubekommen. An der Ekstase des Lappen hatte nach der Meinung unseres Gewährsmannes der Branntwein, den jener zuerst zu trinken begehrt hatte, einen grösseren Anteil als die Magie.

Leider muss ich die in den Reisebericht eingestreuten naturkundlichen Schilderungen und anekdotenhaften Berichte, z.B. über den Vorgänger des Pfarrers in Jukkasjärvi und das in der Kirche aufgestellte Folterrad, übergehen. Ich möchte nur noch zu einigen im Reisebericht vorkommenden lappischen bzw. finnischen Wörtern etwas sagen. Sie können natürlich nicht als "Sprachmaterial" in Frage kommen, da die Schreibweise des linguistisch nicht geschulten, auch nicht linguistisch interessierten Franzosen die Wörter oft fast bis zur Unkenntlichkeit verunstaltet hat. Einige Wörter bezeichnet er ausdrücklich als finnisch wie: leipä, siika, während er kota, jälälä, luppo und merta (> schwed. mjerde), Wörter, die er wahrscheinlich von den finnischen Ruderern oder Wegweisern gehört hatte, für lappisch hält. Von den Lappen hörte er die Unterscheidung des Rentierweibchens (im allgemeinen) ron der milchgebenden Rentierkuh und einige andere Bezeichnungen z.B. für Rentiermilch, Lappenpelz, den Fisch tjuoutja (finn. siika). Irgendetwas hat er wohl von "Verwandtschaft mit dem Griechischen" gehört, denn er möchte das fehlerhaft aufgezeichnete ptalme

\footnotetext{
${ }^{1} \mathrm{Zu}$ diesem Bericht LM's, im besonderen zu der "verkehrt" gehaltenen Trommel sagt E. Manker (op. cit. S. 414), dass die "Verkehrung der Trommel weder vorher noch nachher irgendwo bezeugt ist"... "Motraye erweist sich jedoch im übrigen als zuverlässiger Beobachter und Berichterstatter, so dass es wohl richtiger ist, mit IIallström anzunehmen, dass jener lappische Zauberer sein besonderes Verfahren hatte..."
} 


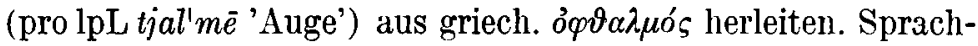
liche Fragen berührt er sonst nicht. ${ }^{1}$

Zum Schluss sei erwähnt, dass Aubry de La Motraye einer der ersten ausländischen Reisenden ist, der Ahvenanmaa besucht und seinen Aufenthalt dort beschrieben hat. Das geschah zwischen dem 9.-13. Sept. 1719, wo er als Begleiter einer zu Friedensverhandlungen mit den Russen aufgebrochenen schwedischen Delegation an dem sog. Alandkongress auf der Insel Vårdö teilnahm und in dem Dorf Lövö (auf LM's Karte Lofvo) wohnte.

\section{JohanNes G. Stipa}

\footnotetext{
${ }^{1}$ Für den Schulunterricht der Lappen wurde LM jedoch so sehr interessiert, dass er, als er den Bericht P. Fjellströms über die guten Erfolge der Skytte-Schule hörte, in seinem Reisebericht den Vorschlag machte, man solle mit einer jährlichen Summe von 100 Talern in jeder Lappmark 10 Schulen einrichten. Den Lapplandbericht samt diesem Vorschlag schickte er gleichsam als Denkschrift an Karl XII., der ihn günstig aufnahm und sogar versprach, ihn drucken zu lassen. Der Tod des Königs am 30. November 1718 vereitelte den Plan.
} 